\title{
Los stakeholders de la industria ho- telera: una clasificación a partir de sus intereses ambientales
}

Stakeholders of the Hotel Industry: A Classification Based on their Environmental Interests

Os stakeholders da indústria hoteleira: uma classificação a partir de seus interesses ambientais

\author{
Yessica Victoria Ortiz ${ }^{\star}$ \\ Elva Esther Vargas Martínez \\ Rosa María Nava Roge ${ }^{* * *}$ \\ Marcelino Castillo Nechar ${ }^{\star \star \star *}$
}

Fecha de recibido: 6 de marzo de 2015

Fecha de aprobado: 25 de septiembre de 2015

Doi: dx.doi.org/10.12804/rev.univ.empresa.30.2016.05

Para citar este artículo: Ortiz, Y. V., Vargas Martínez, E. E., Nava Rogel, R. M. \& Castillo Nechar, M. (2016). Los stakeholders de la industria hotelera: una clasificación a partir de sus intereses ambientales. Universidad \& Empresa, 18(30), 97-120. Doi: dx.doi.org/10.12804/rev.univ.empresa.30.2016.05

* Maestra en Ciencias por el Instituto Politécnico Nacional (México), doctoranda en Estudios Turísticos, Universidad Autónoma del Estado de México. Correo electrónico: yaa260201@ hotmail.com

** Doctora en Ciencias Ambientales por la Universidad Autónoma del Estado de México. Profesora y coordinadora del Centro de Investigación y Estudios Turísticos (Cietur) de la Facultad de Turismo y Gastronomía de la Universidad Autónoma del Estado de México. Correo electrónico: elvacolegio@hotmail.com

*** Doctora en Ciencias Económico-Administrativas por la Universidad Autónoma del Estado de México. Profesora de la Facultad de Contaduría y Administración de la Universidad Autónoma del Estado de México. Correo electrónico: rosanr02@hotmail.com

${ }_{* * * *}^{*}$ Doctor en Ciencia Políticas y Sociales por el Centro de investigación en docencia y Humanidades del estado de Morelos (CIDHEM). Profesor del Centro de investigación y estudios turísticos de la Facultad de Turismo y Gastronomía de la Universidad Autónoma del Estado de México. Correo electrónico: marcanec62@hotmail.com 


\section{RESUMEN}

Esta investigación tiene como fin clasificar a los stakeholders de la industria hotelera que pueden influir en las decisiones organizacionales, en particular, en su responsabilidad ambiental. La clasificación se realiza a partir de los vínculos, los atributos, la preocupación ambiental y la influencia sobre la gestión ambiental de los hoteles. Se utilizó el sistema de Rawlins (2006) y el modelo de atributos y prominencia de Mitchell, Agle y Wood (1997). El estudio posibilita el reconocimiento de los grupos de interés que poseen un alto grado de influencia en materia ambiental, de acuerdo con las características de cada establecimiento hotelero y, una vez establecidos, contribuye a tratar de evitar la ejecución de acciones que puedan agravar la actual crisis ambiental.

Palabras clave: gestión organizacional, grupos de interés, hotelería, intereses ambientales, responsabilidad ambiental, stakeholders.

\section{ABSTRACT}

The purpose of this research is to classify the stakeholders of the hotel industry that, in fact, can influence organizational decisions, in particular, concerning their environmental responsibility. The classification was based on the ties, attributes, environmental concern and influence on the hotel environmental management. The paper uses the Rawlins (2006) system and the attributes and prominence model of Mitchell, Agle y Wood (1997). The study facilitates recognition of stakeholders that exert a high level of influence on environmental matters in accordance with the characteristics of each hotel and, thus, once they are established, help to prevent actions that might increase aggravation of the present environmental crisis.

Keywords: Organizational management, interest groups, hotel industry, environmental interests, environmental responsibility, stakeholders.

\section{RESUMO}

Esta pesquisa tem como fim classificar aos stakeholders da indústria hoteleira que, em efeito, podem chegar a influir nas decisões organizacionais, em particular, em sua responsabilidade ambiental. A classificação se realiza a partir dos vínculos, os atributos, a preocupação ambiental e a influência sobre a gestão ambiental dos hotéis. O trabalho faz uso do sistema de Rawlins (2006) e do modelo de atributos e prominência de Mitchell, Agle e Wood (1997). O estudo faz possível reconhecer os grupos de interesse que possuem um alto grau de influência em matéria ambiental, de acordo com as características de cada estabelecimento hoteleiro e, desta maneira, uma vez estabelecidos, contribui a tentar evitar a realização de ações que possam agravar a atual crise ambiental.

Palavras-chave: gestão organizacional, grupos de interesse, hotelaria, interesses ambientais, responsabilidade ambiental, stakeholders. 


\section{INTRODUCCIÓN}

En su función de construir un nuevo orden social, la globalización ha redistribuido privilegios y despojos, riqueza y pobreza, recursos y desposesión, poder e impotencia (Stiglitz, 2002; 2007). También ha puesto en discusión el progreso económico frente a la falta de responsabilidad de las actividades empresariales (Revenga, 2007). Sin embargo, además de buscar el beneficio económico, las empresas actuales buscan aumentar su valor social y ambiental (Fleming, Roberts \& Garsten, 2013), al asumir una actuación más ética ante aquellos que comparten su trabajo, consumen sus productos o servicios o les dan cabida en su comunidad.

En este marco de gestión empresarial, los negocios se piensan desde una perspectiva diferente, en la que existe, por un lado, la eficiencia económica y, por el otro, una sólida cooperación social basada en la confianza, las normas y una serie de valores compartidos entre los grupos de interés que configuran la organización (Fassin, 2012; Jensen \& Sandström, 2011). Así, el logro de los objetivos y la toma de decisiones se entienden desde la pluralidad de los agentes que la afectan o intervienen en esta y va más allá del contrato jurídico o social existente entre ellos.
Hoy en día, el sistema de relaciones económicas, políticas y sociales permite analizar los vínculos entre la empresa y los agentes, uno de los cuales está encaminado a superar el abuso de las organizaciones y el bienestar particular, para alcanzar un bienestar social y sustentable (Aguinis \& Glavas, 2012).

La industria turística es una de las actividades económicas que, durante las últimas décadas, ha tenido un crecimiento significativo (Ioannides \& Debbage, 1998), pues las cadenas hoteleras trasnacionales ocupan un lugar determinante tanto en las redes de flujos financieros, tecnológicos y demográficos como en el uso y la apropiación de recursos naturales; por ello, es importante reconocer cómo la teoría de los stakeholders puede ayudar a explicar el comportamiento empresarial respecto a las cuestiones ambientales.

Este artículo, que revisa la literatura relativa a los aspectos teóricos (vinculados con los discursos que aborda el trabajo) y a los prácticos (referentes a la industria hotelera), tiene el propósito de identificar a los stakeholders de las empresas hoteleras y determinar cómo estos se relacionan con la responsabilidad ambiental de las mismas.

En cuanto a la metodología, se hace un análisis que aplica el modelo de 
Rawlins (2006) y define los grupos de interesados en la hotelería. Asimismo, con base en el modelo de atributos y prominencia de Mitchell y otros autores (1997), se identifican los stakeholders de las empresas hoteleras de acuerdo con tres atributos esenciales: urgencia, legitimidad y poder. Luego, se categorizan los stakeholders según su conocimiento y preocupación por la situación ambiental y aquellos que prestan mayor atención a dicha problemática. Por último, a partir del trabajo realizado, se propone una clasificación de los stakeholders asociados con las empresas de hospedaje, a partir de las acciones que ejecutan en favor del ambiente y de la influencia que ejercen sobre la organización y su responsabilidad ambiental.

\section{TEORÍA DE LOS STAKEHOLDERS}

El término stakeholder ${ }^{l}$ comenzó a popularizarse a partir de la década del cincuenta y del sesenta, cuando la empresa otorgó importancia a los agentes que se relacionaban con ella. En un inicio, los stakeholders se definieron como "aquellos grupos sin cuyo apoyo la organización podría dejar de existir" (González, 2012, p. 74). Más adelante, el término adquirió una definición más clara, al interpretarse como cualquier grupo o individuo que pudiera afectar o ser afectado por el logro de los propósitos de una corporación (Freeman, 2010; Parmar et al., 2010; Phi1lips \& Freeman, 2010).

El concepto de stakeholder se distingue entre una acepción restringida y otra más amplia. Por una parte, se limita a los grupos o individuos que se relacionan con la organización y, por otra, se concibe como todos los grupos o individuos de los cuales depende la supervivencia empresarial, ya que adoptan un papel de interlocutores válidos que pueden influir en las decisiones y actividades de la compañía, por lo que son considerados como agentes estratégicos capaces de promover la creación de riqueza e impulsar el crecimiento a largo plazo.

Entender la relación entre la organización y sus stakeholders ha sido útil para comprender toda una plataforma de aprendizaje, comunicación, compromiso, innovación y mejora en los resultados económicos de la organización. Además, también ha permitido explicar el comportamiento empresarial alrededor de cuestiones sociales, puesto

1 Este término se halla en la memoria que el Stanford Research Institute (SRI) elaboró en 1963 sobre el concepto de empresa. 
que se identifican los intereses y las necesidades de los actores involucrados con la organización por medio de los vínculos que se establecen entre empresa e interesado.

Responder a los requerimientos de los grupos de interés no implica complacer todas sus peticiones, sino crear un mecanismo de diálogo y entendimiento mutuo que considere las necesidades para la toma de decisiones de la organización y se utilice como instrumento mediador para la resolución de conflictos y la generación de estrategias (Habermas, 1989).

A partir de un enfoque ético en estas relaciones, surge el reconocimiento de una cierta responsabilidad hacia los otros, de un compromiso con los demás, como una acción en forma de ayuda que es motivada por una solicitud cuyo fin último es lograr el cuidado de los semejantes o de sí mismo (Lawrence \& Weber, 2011). Esta postura ética supone una preocupación por la posibilidad de omisión, de no ayudar cuando es posible hacerlo (Frederick, Post \& Davis, 1992). Sin embargo, lo que se busca no es solo contener la agresión, la falta de respuesta o de acción cuando habría que hacerlo, sino contribuir a un mejor desempeño moral de la empresa, al desarrollar la cooperación, la compasión y el cuidado en todas las áreas de la misma. Esto puede contribuir a su éxito como negocio (Albizu \& Landeta, 2001; Phillips, 2003).

La literatura relativa al comportamiento social de la empresa, en particular el trabajo de Wood (1991), por ejemplo, permite identificar la existencia de criterios vinculados con el rendimiento ambiental de la organización (Ilinitch, Soderstrom \& Thomas, 1999). Se considera que la teoría de los grupos de interés también es capaz de explicar - y es necesario que lo haga - el comportamiento empresarial alrededor de las cuestiones ambientales (Orts \& Strudler, 2002).

La presión que ejercen los stakeholders puede convertirse, de algún modo, en el origen de las respuestas de las empresas (Henriques \& Sadorsky, 1999). Es importante para la agenda de investigación identificar quiénes son los stakeholders más representativos en materia ambiental para los establecimientos de hospedaje, pues, como mencionan Fineman y Clarke (1996), Rodríguez y Ricart (1997) y Stead y Stead (2000), una vía adecuada de canalizar la presión social hacia la protección ambiental consiste en el análisis de los principales grupos de interés afectados por la empresa. 
En el campo de la responsabilidad ambiental, se requiere una atención focalizada en la gestión que hace la empresa hacia la conservación de la naturaleza, para visualizar representaciones de racionalidad más amplias para los stakeholders. En este proyecto, se busca presentar un enfoque amplio e integral de la ética empresarial y su dimensión ambiental, dentro del marco general de un modelo de stakeholders, porque es uno de los mejores esquemas disponibles para entender y articular estas importantes cuestiones.

\section{IDENTIFICAR LOS STAKEHOLDERS}

En la actividad turística, poco se ha trabajado para identificar a los stakeholders y su interés en la protección empresarial a la naturaleza. A pesar de esto, los estudios existentes proporcionan diversas perspectivas para hacerlo. Así, en el intento de de- terminar los stakeholders clave para la industria de la hotelería y tratar de gestionarlos, en atención a necesidades e intereses en materia ambiental, se utilizó el modelo sistemático e integral desarrollado por Rawlins (2006). Este modelo consta de cuatro pasos consecutivos que priorizan aquellos stakeholders a quienes debieran destinarse los esfuerzos de diálogo y correspondencia para construir la dimensión ambiental de las empresas hoteleras (figura 1):

1. Identificar todos los posibles stakeholders por su relación con establecimientos de hospedaje.

2. Priorizar los stakeholders de acuerdo con sus atributos.

3. Priorizar los stakeholders de acuerdo con la situación ambiental.

4. Priorizar los stakeholders de acuerdo con la influencia que ejercen en la gestión ambiental de las empresas.

Figura 1. Modelo de gestión de vínculos con los stakeholders

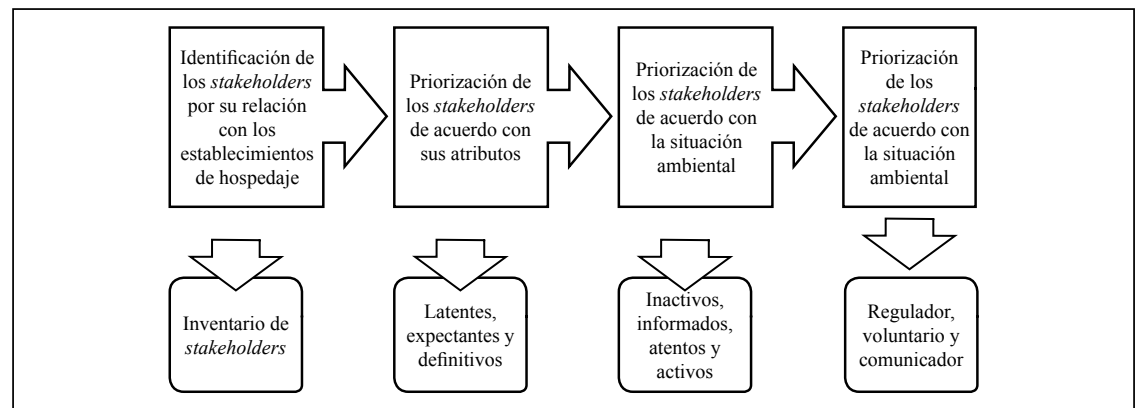

Fuente: elaboración propia con base en Rawlins (2006). 


\section{IDENTIFICACIÓN DE LOS STAKEHOLDERS POR SU RE- LACIÓN CON LOS ESTABLECI- MIENTOS DE HOSPEDAJE}

El citado modelo plantea la existencia de cuatro tipos de vínculos que identifican las relaciones de las partes interesadas con una organización. Estos son: 1) de apoyo o posibilitadores; 2) funcionales; 3) normativos, y 4) difusos.

Los vínculos de apoyo o posibilitadores permiten identificar a los stakeholders que tienen control o autoridad sobre la organización y proveen recursos financieros u otorgan permisos para que los hoteles puedan operar. Si la relación se complica, es posible que estos apoyos queden limitados, lo que dejaría a la empresa en imposibilidad de maniobrar. Si, por lo contrario, es fluida, completa y veraz, se contribuye a crear un nexo eficiente para el proceso de toma de decisiones.

Los vínculos funcionales son esenciales para la operación de la empresa hotelera. Puesto que los destinos turísticos son pensados como un clúster y están integrados por elementos abastecedores (inputs) y elementos que salen de la actividad (outputs), en los hoteles se organizan cadenas productivas de diversa naturaleza (Vargas, López \& Cer- vantes, 2014). Los vínculos funcionales que se ejercen con la organización se dividen entre las funciones de entrada - que proporcionan la mano de obra para crear $u$ ofrecer un producto o servicio- y las de salida — que sirven para el consumo o comercialización del mismo-.

Los vínculos normativos pueden generarse por dos vías: la reguladora y la voluntaria. Los stakeholders de este tipo inciden directamente en la estandarización de las actividades de la organización, representados por algunas instituciones gubernamentales o asociaciones con actividades similares. Las relaciones se establecen de acuerdo con acciones, propósitos o problemas de carácter común como la promoción turística, la capacitación, la calidad en el servicio o la responsabilidad social. Estas pueden llegar a permitir la realización de análisis comparativos, así como de asesorías o certificaciones.

Los vínculos difusos incluyen todos aquellos stakeholders con los que no se tiene una constante interacción como la comunidad, las ONG o las organizaciones civiles y pueden revalorizarse en tiempos de conflicto o crisis. Ante los problemas sociales y ambientales que enfrentan los destinos turísticos, por ejemplo, por la presencia de grandes cadenas hoteleras, las relaciones se tornan proble- 
máticas. En estos casos, es factible establecer vínculos con medios de comunicación, grupos ambientalistas o comunidades indígenas para tratar de resolver los conflictos y beneficiarse entre sí.

Una empresa hotelera está inmersa en un sistema en constante corres- pondencia con la comunidad en la que desarrolla sus actividades. Por ello, el vínculo que surge entre ambas provoca que estas pasen a ser miembros activos de la sociedad y deban estar atentas a cumplir con las demandas de la población y a generar una conexión cada vez más fortalecida y consolidada (figura 2).

Figura 2. Vínculos de las empresas hoteleras con los stakeholders

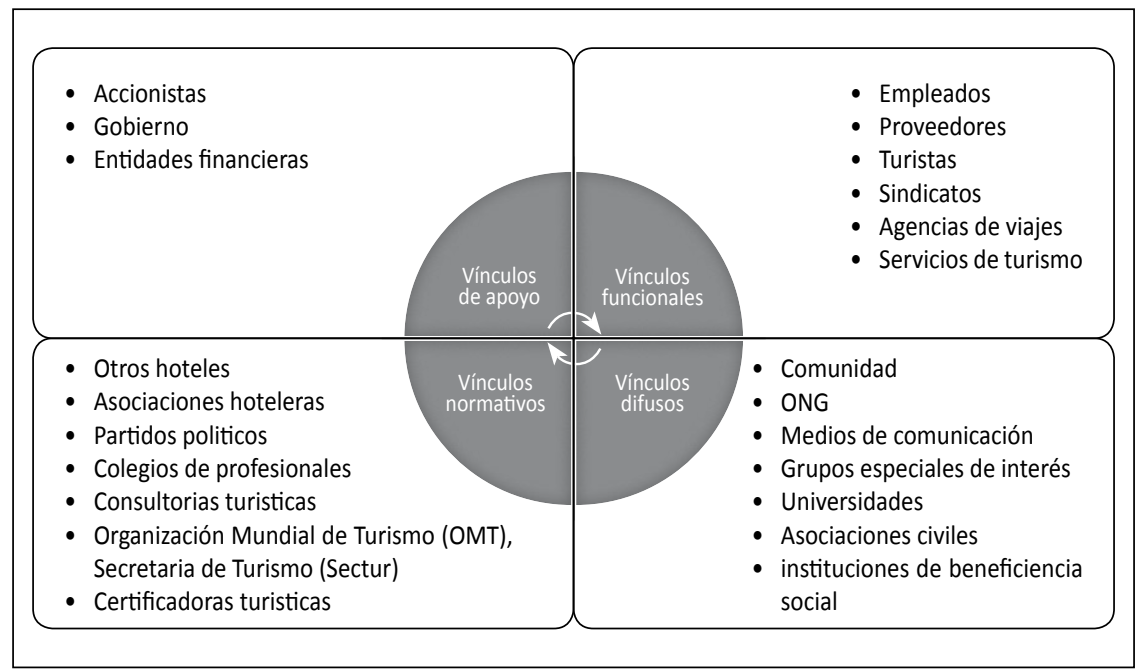

Fuente: elaboración propia.

En la teoría ética, entra en acción el diálogo entre las partes para la búsqueda de consensos acerca de las normas y los procedimientos empresariales que puedan responder a intereses "universalizables" que guíen la dirección empresarial (González, 2012).

Por medio del diálogo se da a conocer cuáles son los intereses de cada stakeholder y de la propia organi-

2 Son los intereses que pueden compartirse por todos los stakeholders afectados por la estrategia empresarial. 
zación. Además de tener intereses a corto plazo, como la obtención de recursos financieros, las empresas tienen objetivos de largo plazo, encaminados a su permanencia en el mercado. Para satisfacer dichos intereses, surgen diversas relaciones de la empresa con sus stakeholders, entre las que sobresale aquella con el trabajador, ya que existe una codependencia de intereses. Al impulsar puestos de trabajo, los empresarios hacen necesaria la inclusión de empleados a la organización para el desarrollo de las actividades empresariales $\mathrm{y}$, de este modo, los empleados esperan obtener una retribución económica por las acciones realizadas.

Para categorizar los stakeholders por su relación con la empresa hotelera, no solo es necesario satisfacer el interés particular o grupal con la actividad empresarial, sino que debe considerarse al actor o grupo de actores como un interlocutor válido, como una instancia que influye en los actos de la organización, de acuerdo con la función que este desempeña en cada momento. El diálogo con los stakeholders puede clasificarse en cuatro grupos (Raufflet, Lozano \& De la Torre, 2012):

1. Stakeholder comunicacional: los participantes en el diálogo aceptan que no utilizarán sus recursos a manera de manipulación, sino de obligación y derechos.
2. Stakeholder inclusivo: incluye a todos los stakeholders potenciales, presentes y futuros, para participar en los diálogos. Se estipula que ningún stakeholder puede ser excluido de la participación.

3. Stakeholder participativo: se estipula que los stakeholders deben desarrollar el diálogo en condiciones de igualdad de oportunidades, de participación y en consideración de las posibilidades de intervención de los actores en los intereses en juego.

4. Stakeholder relacional: todos los intereses deben ser considerados por igual y estar abiertos a revisión basada en razones. Por este motivo, ningún interés debe ser pensado como definitivo y libre de crítica.

Como se observa, el criterio del diálogo es clave para una buena gestión de los grupos de interés, debido al valor instrumental que posee y que representa un enfoque ético-normativo para la empresa.

\section{PRIORIZACIÓN DE LOS STAKEHOLDERS DE ACUERDO CON SUS ATRIBUTOS}

Mitchell, Agle, Chrisman y Spence (2011) aseguran que dentro de la literatura de la teoría de los stakeholders no se encuentran atributos específicos que orienten de manera 
fiable una categorización de las partes interesadas (Agle, Mitchell \& Sonnenfeld, 1999). Después de una amplia revisión, estos autores determinan tres atributos que contribuyen a su identificación: urgencia, legitimidad y poder. Los investigadores señalan que, sobre la base de la posesión de uno, dos o tres de ellos, es posible definir el campo de los interesados.

Se dice que los stakeholders tienen urgencia cuando sus demandas requieren una atención inmediata y son críticas. La urgencia, por sí misma, no puede determinar la jerarquía de un stakeholder, pero no debe ser ignorada, puesto que suele atraer la atención de otras partes del clúster.

La legitimidad, en términos generales, se refiere a la opinión generalizada de los integrantes de un sistema social en donde las acciones de personas u organizaciones son deseables, apropiadas y acordes con las normas, los valores, las creencias y las definiciones propias de dicho sistema social. Ledingham (2009) menciona que, para que un stakeholder sea legítimo, debe establecerse un vínculo. Así, de una gestión adecuada de los vínculos, con fundamento en intereses y metas compartidos, también resulta una administración adecuada de stakeholders.
Frooman (1999) se centra en el concepto de poder como un componente estructural de la relación entre las empresas y los grupos de interés. Este autor se apoya en la teoría de dependencia de recursos (Pfeffer \& Salancik, 1978). Indica que los stakeholders se han convertido en controladores de recursos de la organización, a causa del poder de influencia que desarrollan sobre ellas, por lo que las compañías muestran cierto interés por identificar las estrategias más aptas para aproximarse a las expectativas de sus stakeholders.

Es importante resaltar que el poder es tal vez más difícil de definir que de reconocer. Los stakeholders lo tienen cuando inciden en las decisiones organizacionales, cuando exigen a una organización hacer algo que, sin la presencia de este requerimiento, ella no hubiera hecho por su cuenta. Mintzberg (1983) describe el poder como la capacidad para persuadir en el logro de los resultados organizacionales. Los stakeholders lo utilizan para influir en el comportamiento de quienes están en capacidad de contribuir a la consecución de ese logro y también para tratar de satisfacer sus necesidades e intereses en cuanto a la compañía.

La parte más importante dentro de esta postura es que no todos los stakeholders buscan cumplir las mis- 
mas metas ni obtener los mismos beneficios. En algunos casos, existen intereses contradictorios, los cuales, en no pocas ocasiones, generan conflictos entre los stakeholders y la empresa (Azzone, Brophy, Noci, Welford \& Young, 1997; Grafé Buckens \& Hinton, 1998; Johnson, 1998).

Según el modelo de atributos y prominencia, elaborado por Mitchell y otros autores (1997), los stakeholders que poseen uno solo de los atributos tienen poca prominencia y deben ser considerados como latentes; aquellos que posean dos atributos tienen prominencia media y deben ser considerados como expectantes $\mathrm{y}$ quienes tienen los tres atributos obtienen gran prominencia y deben ser considerados como definitivos.

La presencia de uno de los atributos anteriores o la combinación de estos origina una subcategoría identificada por Mitchell y otros autores (1997) y se describe en la tabla 1.

Se considera que los stakeholders que no ostenten alguno de los tres atributos no son, en realidad, stakeholders legítimos.

Tabla 1. Descripción de atributos de los stakeholders

\begin{tabular}{|c|c|c|c|}
\hline Categoría & Atributo & Denominación & Definición \\
\hline \multirow{3}{*}{$\begin{array}{l}\text { Latentes } \\
\text { (poseen un } \\
\text { atributo) }\end{array}$} & Poder & Inactivos & $\begin{array}{l}\text { Poseen poder, pero no exigencia o un vínculo le- } \\
\text { gítimo con el hotel, lo que les impide hacer valer } \\
\text { su poderío. } \\
\text { Están dispuestos a adquirir un segundo atribu- } \\
\text { to para imponer su voluntad. Un ejemplo de esta } \\
\text { categoría son los grupos con poder coercitivo o } \\
\text { económico, también los que constituyen agentes } \\
\text { amenazantes (p. ej., la prensa). }\end{array}$ \\
\hline & Legitimidad & Discrecionales & $\begin{array}{l}\text { Su relación con la organización se mueve en un } \\
\text { ámbito filantrópico. Dado que no cuentan con } \\
\text { poder ni demandas urgentes para satisfacer, solo } \\
\text { mantienen una relación legítima con el hotel. En } \\
\text { esta categoría caben las fundaciones, las escuelas } \\
\text { o los grupos de ayuda social. }\end{array}$ \\
\hline & Urgencia & Demandantes & $\begin{array}{l}\text { La relación entre los stakeholders demandantes y } \\
\text { la organización surge a raíz de una demanda por } \\
\text { parte del stakeholder; sin embargo, dicho grupo en } \\
\text { esta clasificación no necesariamente tiene la fuerza } \\
\text { suficiente o el reconocimiento social para que su } \\
\text { demanda sea atendida. }\end{array}$ \\
\hline
\end{tabular}

Continúa 


\begin{tabular}{|l|c|c|l|}
\hline Categoría & Atributo & Denominación & \multicolumn{1}{c|}{ Definición } \\
\hline $\begin{array}{l}\text { Poder y } \\
\text { legitimidad }\end{array}$ & Dominantes & $\begin{array}{l}\text { Debido a la legitimidad y poder que poseen estos } \\
\text { stakeholders, al manifestar sus intereses, expec- } \\
\text { tativas y demandas, la organización las considera } \\
\text { importantes; no obstante, al carecer de urgencia, su } \\
\text { atención no suele ser inmediata. }\end{array}$ \\
\cline { 2 - 5 } $\begin{array}{l}\text { Expectantes } \\
\text { (poseen dos } \\
\text { atributos) }\end{array}$ & $\begin{array}{c}\text { Poder y } \\
\text { urgencia }\end{array}$ & Peligrosos & $\begin{array}{l}\text { Su relación con la organización puede ser coerci- } \\
\text { tiva, debido a que puede ser impuesta con el uso } \\
\text { del poder o de la fuerza. Al carecer de legitimidad, } \\
\text { no tienen la facultad de exigir a la organización un } \\
\text { determinado comportamiento. }\end{array}$ \\
\cline { 2 - 4 } & $\begin{array}{l}\text { Legitimidad } \\
\text { y urgencia }\end{array}$ & Dependientes & $\begin{array}{l}\text { Al carecer de poder, dependen de otros grupos de } \\
\text { interés internos o externos para lograr que sus de- } \\
\text { mandas sean atendidas por el hotel. En este caso } \\
\text { se puede encontrar la comunidad, debido a que, en } \\
\text { ocasiones, ella debe recurrir a instancias guberna- } \\
\text { mentales para que sus demandas sean atendidas. }\end{array}$ \\
\hline $\begin{array}{l}\text { Definitivos } \\
\text { poseen tres } \\
\text { atributos) }\end{array}$ & $\begin{array}{c}\text { Pogitimidad } \\
\text { y urgencia }\end{array}$ & Prioritarios & $\begin{array}{l}\text { Dentro de este grupo se encuentran los stakehol- } \\
\text { ders más representativos para la organización. Tie- } \\
\text { nen un alto impacto en las decisiones de la empresa } \\
\text { y sus demandas son las primeras en ser atendidas. }\end{array}$ \\
\hline
\end{tabular}

Fuente: elaboración propia con base en Mitchell y otros autores (1997).

La identificación de stakeholders según sus atributos puede ser cambiante, pues nunca tienen un comportamiento estático. Aunque la clasificación anterior muestra un comportamiento constante, este puede transformarse para cada hotel y en períodos indefinidos.

\section{IDENTIFICACIÓN DE}

LOS STAKEHOLDERS DE ACUERDO CON LA SITUACIÓN AMBIENTAL

La preocupación de ciertos grupos por mejorar los problemas ambientales está asociada con una gran can- tidad de aspectos y enfoques. Desde el campo sociológico, se enfatiza en los movimientos ecológicos, los riesgos tecnológicos o las políticas ambientales; la Psicología, por su parte, se orienta a las actitudes, los valores y las conductas de grupos o individuos para enfrentar esta problemática.

Frente al crecimiento turístico y al desarrollo de nuevos destinos, también surge el interés por una nueva conciencia ambiental de los consumidores y su atención al cuidado de la naturaleza. Se preservan paisajes y ecosistemas que son vistos como 
atractivos naturales, tras un proceso de valorización social que refleja la tendencia y el gusto particular de ciertos sectores de la sociedad (Kuper, 2009).

La demanda social por la solución a problemas ambientales tiene que ver con la magnitud de la molestia generada, por el daño que provoca y por el potencial peligro que representa para la comunidad. Asimismo, se argumenta que el incremento en la destrucción de la naturaleza no es lo que explica la emergencia de la conciencia ambiental de los individuos, puesto que, para que la sociedad demuestre preocupación, intervienen factores culturales, sociales y políticos. Ante la enorme cantidad de cambios en la vida pública, los intereses y las demandas por el ambiente se van modificando y aparecen nuevos tópicos.

Lezama (2004) alude al proceso por el cual se construyen las demandas ambientales. Hannigan (1997), por su parte, identifica tres condiciones: el montaje del problema, la presentación y la discusión o el debate de las demandas. El montaje equivale a la valoración e identificación de aquellos agentes responsables de tomar medidas correctivas para la solución de un problema ambiental. Generalmente se ubica entre las personas que tienen conocimiento y habilidades para comprenderlos y resolverlos de una manera más efectiva. En cuanto a la presentación, se pueden reconocer aquellos grupos que atraen la atención a partir de una manifestación original y entendible de la situación ambiental que se vive. Por último, la discusión o el debate de las demandas se sustenta en el hecho de que los problemas ambientales también representan problemáticas sociales que agravian a las personas; por ello, se buscan agentes que puedan presionar a las organizaciones, instituciones o entidades responsables, para generar un cambio.

Para complementar lo anterior, Lezama (2004) considera otro planteamiento que clasifica el proceso de las demandas ambientales en tres partes: las demandas mismas, aquellos quienes las plantean y su proceso de diseño (Best, 1987). Desde esta perspectiva, se reconoce la manera en la que los problemas ambientales son llevados a la atención pública y el grado de compromiso que las personas tienen con estos. Las propias demandas representan las quejas o molestias planteadas por un grupo y cómo son presentadas para persuadir a su audiencia. Respecto a quienes las plantean, se trata de la identificación de personas facultadas para hablar, como científicos, políticos, académicos y autoridades con enten- 
dimiento del tema. El diseño de demandas alude a los grupos que legitiman la situación ambiental que se vive y argumentan su existencia, como lo hacen los medios de comunicación.

En este marco de consideraciones, el grado de interés por el cuidado de la naturaleza se asocia con el conocimiento de la problemática ambiental. En esto existe un abismo entre lo conocido y la realidad: una sociedad ignorante de la problemática ambiental, ¿está condenada a sufrir las consecuencias de dicha crisis? Hajer (1995) manifiesta que la parte política desempeña un papel determinante en esta situación, una política que no solo sea creada como mecanismo para encontrar soluciones a problemas ambientales preconcebidos, sino también como una forma práctica para que la sociedad pueda resolver los problemas sociales resultantes.
MacNaghten y Urry (2001) afirman que el interés por la protección del ambiente no suele tener la misma intensidad en todas las esferas sociales. La población más afectada por los daños ambientales no lucha con la misma intensidad que aquella en la que las normas de salud, seguridad y bienestar están más consolidadas. Además, se puede afirmar que el grado de conciencia ambiental y la protesta no están siempre relacionados, debido a los cambios en las prácticas sociales que originan distintas actitudes y preocupaciones por la naturaleza.

Desde la perspectiva de Hallahan (2001), los stakeholders pueden categorizarse por su grado de conocimiento ambiental, del problema o de su causa y también por su grado de implicación. De esto último depende el interés, la preocupación y la presión que puedan ejercer sobre determinadas entidades (tabla 2).

Tabla 2. Jerarquización de los stakeholders de acuerdo con la situación ambiental

\begin{tabular}{|l|l|}
\hline Categorización & \multicolumn{1}{c|}{ Características } \\
\hline Inactivos & $\begin{array}{l}\text { Dentro de esta categoría se encuentran los stakeholders con escaso conocimiento } \\
\text { y poca implicación en la problemática ambiental. El tema ambiental es un aspecto } \\
\text { que no interviene en las decisiones que se toman en relación con su vida. }\end{array}$ \\
\hline Atentos & $\begin{array}{l}\text { Este tipo de stakeholders tiene escaso conocimiento de la problemática ambiental; } \\
\text { sin embargo, reconoce que existe el problema y, en consecuencia, se mantiene atento. }\end{array}$ \\
\hline Informados & $\begin{array}{l}\text { Constituyen los grupos que tiene bastante conocimiento de la problemática am- } \\
\text { biental; no obstante, mantienen poca implicación en cuanto a la organización. } \\
\text { La relación entre ambos puede presentarse de manera voluntaria, en beneficio } \\
\text { de uno o de otro. }\end{array}$ \\
\hline
\end{tabular}




\begin{tabular}{|l|l|}
\hline Categorización & \multicolumn{1}{c|}{ Características } \\
\hline Activos & $\begin{array}{l}\text { Este grupo de stakeholders es el que puede tener un mayor impacto en temas am- } \\
\text { bientales, debido a que hay bastante conocimiento e implicación en la problemá- } \\
\text { tica ambiental. Los grupos más fuertes en esta categoría suelen ser las autoridades } \\
\text { gubernamentales y los ambientalistas o defensores sociales, ya que tienen mayor } \\
\text { influencia para provocar un cambio. }\end{array}$ \\
\hline
\end{tabular}

Fuente: elaboración propia con base en Hallahan (2001).

\section{IDENTIFICACIÓN DE LOS STAKEHOLDERS DE ACUERDO CON SU INFLUENCIA EN LA RESPONSABILIDAD AMBIENTAL DE LOS HOTELES}

Durante los últimos años, la degradación ambiental ha generado un interés manifiesto por el cuidado y la protección de la naturaleza. La presión que ejercen los stakeholders sobre las empresas puede convertirse en el origen de respuestas ambientales (Henriques \& Sadorsky, 1999). De este modo, identificar quiénes son los grupos más representativos constituye una importante gestión que deriva en un comportamiento em- presarial más responsable (Gilbert, Stead \& Ivancevich, 1999; Henriques \& Sadorsky, 1999; Rodríguez \& Ricart, 1997).

Entre los múltiples intentos de clasificar estas partes se encuentra el de Baro (2011). Este autor señala la existencia de diversos mecanismos ${ }^{3}$, entre los cuales el grado de influencia que ejerce sobre la organización es uno de los más relevantes. Ante el ejercicio de esta presión y su respuesta, los grupos pueden disminuir, retrasar, suspender o anular sus relaciones con la empresa y desencadenar un enfrentamiento con la misma, al desplegar su podero buscar

3 Algunos estudios mencionan a un primer grupo que enfatiza el rol de los stakeholders externos en la valoración del rendimiento y el riesgo ambiental (Henriques \& Sadorsky, 1996, Klassen \& Vachon, 2003, Sharma \& Henriques, 2005). Otros autores, como Azzone, Brophy, Noci, Welford y Young, 1997, Tilt (1997) y Thomas (1992), buscan demostrar la importancia de los grupos de interés para que las organizaciones incluyan en sus actividades programas de comunicación e información ambiental. Agle, Mitchell y Sonnenfeld (1999), Buysse y Verbeke (2003), Céspedes, De Burgos y Álvarez (2003) y Fernández y Nieto (2002) identifican a los stakeholders más relevantes en relación con las estrategias ambientales de la organización. Paralelamente, se consideran los impactos ambientales que surgen de la actividad de la empresa hacia determinados stakeholders, lo cual es visto por numerosos autores como un factor clave para la supervivencia de la misma (Berry \& Rondinelli, 1998; Fineman \& Clarke, 1996; Rodríguez \& Ricart, 1997; Stead \& Stead, 2000; Welford \& Gouldson, 1993). Delmas y Toffel (2004), Collins y Usher (2004), Handfield, Sroufe y Walton (2005) y Polonsky, Rosenberger y Ottman (1998) enfocan sus estudios a la cooperación entre empresa y los stakeholders para el cuidado ambiental. 
aliados que puedan ejercerlo (Hill \& Jones, 1992).

En este caso, la definición que se ha hecho de los stakeholders como aquellos grupos o individuos que tienen un interés en las acciones de la organización y son capaces de influir en ella recobra sentido. En los últimos años, las empresas hoteleras han adoptado ciertos comportamientos a favor del ambiente, a causa de la influencia de sus stakeholders. De acuerdo con la teoría de las partes interesadas y la literatura ambiental, los grupos de interés pueden expresar su voluntad e influir en la gestión de los hoteles por medio de la presión directa o por la transmisión de información. Se identifican tres grupos: 1) los interesados reguladores; 2) los voluntarios, y 3) los comunicadores.

Los stakeholders reguladores serán capaces de convencer a los hoteles para implementar la gestión ambiental, normalizar sus procesos de operación e incluir tecnologías ambientales. Existen reguladores externos e internos. Los primeros influyen para que las empresas hoteleras se integren a un marco regulador de protección ambiental que todos los hoteles deberían cumplir (Gobierno, autoridades ambientales, grupos ambientalistas, comités verdes y competidores de las mismas empresas).
Los segundos impactan en la adopción de la gestión ambiental en el hotel mediante una influencia directa (clientes, empleados, proveedores $\mathrm{y}$ accionistas).

El Gobierno suele exigir a los hoteles el cumplimiento de requisitos ambientales legales desde el momento de su construcción y durante el desarrollo de sus actividades y hace inspecciones para verificar su responsabilidad ambiental, por lo que los hoteles asumen la gestión ambiental para mantenerse blindados contra inspecciones, multas y sanciones (Vargas, Zizumbo, Viesca \& Del Carmen Serrano, 2011). Las organizaciones internacionales, las asociaciones hoteleras y los grupos ambientalistas son fuente de importantes sistemas de gestión ambiental y certificaciones de los hoteles, así como los competidores que obligan a sus rivales a mantener un liderazgo ambiental dentro del sector turístico (Barret, 2002).

En materia ambiental, los grupos que mayor presión ejercen sobre las empresas hoteleras son los clientes y los operadores de turismo. La influencia surge por el tipo de actividades que realiza el huésped durante su estancia y el contacto que establece con los recursos naturales. A esto se suma el hecho de que los clientes responden positivamente a 
la selección de un hotel que muestre cuidado por el ambiente por medio de la reservación del servicio (Álvarez, De Burgos \& Céspedes, 2001; Llul, 2001). También puede sentirse satisfecho por las acciones de gestión ambiental que observe durante su estancia, como prácticas de ahorro de agua y energía.

En ocasiones, los accionistas, como uno de los stakeholders de los hoteles, son los más interesados en un adecuado manejo ambiental por parte de la organización, a causa de los beneficios que pueden obtener al mostrar un comportamiento verde (Miller \& Szekely, 1995). La importancia de integrar la gestión ambiental a la estructura organizacional de los hoteles se percibe en tres sentidos por parte de los directivos: como una posibilidad de que la empresa sea más rentable, de que mejore su imagen corporativa y de que trate de incidir, con sus acciones, en la estructura del sector turístico, a fin de ganar poder y posicionamiento en el mercado (Dief \& Font, 2010; Vargas et al., 2011).

Los empleados representan un importante elemento dentro de la operación del servicio turístico $\mathrm{y}$, por lo tanto, pueden ejercer una fuerte influencia en el ejercicio de la política ambiental. En hoteles en donde se opera con buenos desempeños en esta materia, los empleados perciben a la empresa como una compañía reconocida, que mantiene buena imagen ante sus clientes y que cuenta con valores y respeto por la naturaleza. Por este motivo, suelen sentirse orgullosos de trabajar en ella (Vargas et al., 2011).

Los grupos de proveedores pueden presionar a un hotel si este no cumple con los estándares de calidad y el adecuado y esperado uso racional de los productos. Si el proveedor ve amenazada su reputación, puede sustituirlo por otro cliente.

Dentro de los grupos voluntarios se encuentra la comunidad. Esta puede movilizar a la opinión pública sobre el comportamiento de los hoteles. Las protestas de los residentes sobre contaminación por parte de estas organizaciones, en diversos destinos turísticos, es una de las más importantes razones para que los establecimientos mejoren su actuación.

En la última categoría están los medios de comunicación (la prensa, la televisión, la radio y las redes sociales). El público está cada vez más informado sobre la crisis ambiental por estos medios, de modo que, casi de inmediato, puede obtener el reporte de las catástrofes ambientales que suceden en ciertos destinos turísticos y si estas son atribuibles a 
empresas hoteleras. Así, su influencia se deriva, en particular, de la información que ofrecen en relación con las empresas (Henriques \& Sadorsky, 1999).

\section{IMPLICACIONES PARA LA GESTIÓN HOTELERA}

Las empresas pueden atender las demandas de los stakeholders por dos razones: 1) por el valor intrínseco (enfoque normativo), de manera que asumirlas supone legitimar su actuación frente a la sociedad, y 2) puesto que puede mejorar la rentabilidad de la empresa (enfoque instrumental) (Donaldson \& Preston, 1995).

Existen algunas acciones que los hoteles adoptan para ser "ambientalmente responsables". La adopción de prácticas ambientales es una acción que busca reducir el impacto negativo que causan los procesos productivos, por medio de modificaciones efectivas en la organización de los mismos y en las actividades que los componen.

Las empresas hoteleras se apoyan en diversos procesos para minimizar los impactos de sus actividades sobre la naturaleza. Algunas lo hacen con la adopción de sistemas de gestión ambiental, otras buscan obtener diversas certificaciones en esta materia y algunas incorporan la idea del consumo responsable dentro de sus acciones prioritarias. De la adopción de estrategias ambientales surge la última clasificación de los stakeholders involucrados con los establecimientos de hospedaje.

A manera de conclusión, se puede decir que los clientes, el Gobierno y los operadores turísticos son los stakeholders que ejercen mayor presión sobre los hoteles para que desarrollen sus prácticas con una visión de responsabilidad ambiental. Como ya se ha indicado, en ocasiones, son los propios directivos de las empresas quienes suelen anticiparse a las demandas que estos stakeholders podrían hacer a la organización.

Es fundamental tener en cuenta que las empresas actúan de modos distintos. Algunas se resisten al cambio, mientas otras procuran adaptarse a las necesidades del mercado e integrar estrategias ambientales que les faciliten una mejor relación con sus grupos de interés. Por esto, los resultados que se obtienen también son diferentes, ya que, en algunos casos, se busca el cambio de manera voluntaria y, en otros, por la presión de los stakeholders.

\section{CONCLUSIONES}

En este trabajo se hizo un análisis aplicativo del modelo de Rawlins (2006) a la hotelería y la responsa- 
bilidad ambiental. Cabe recordar que, hoy en día, es esencial que las empresas conozcan y se relacionen con sus stakeholders, debido a que ellos pueden afectar negativa o positivamente los procesos de la organización.

La participación de los stakeholders constituye un aspecto novedoso que otorga grandes ventajas para las organizaciones. Luego de identificarlos con claridad, la organización debe conocer cuáles son sus necesidades, expectativas y demandas, con el fin de atender estos aspectos en sus procesos decisorios.

En cuanto a la actividad turística - en particular, al sector hotelero-, el establecimiento de los stakeholders para analizar la situación ambiental que los rodea es determinante. Como se observó en este trabajo, existe una serie de factores que puede dar una mayor relevancia a determinado stakeholders respecto a otro.

Al conocer a los stakeholders que podrían ejercer una mayor presión sobre los hoteles, estos pueden propiciar un acercamiento a tales grupos, con la finalidad de conocer sus demandas y considerarlas durante la toma de decisiones. Esto puede ser determinante para la permanencia de un hotel en el mercado competitivo; sin embargo, las empresas no deben pretender un trabajo conjunto con sus stakeholders solo para mantenerse en el mercado, sino para favorecer la protección del ambiente.

Para efectos de esta investigación, se hizo un análisis exclusivo de los stakeholders de las empresas de hospedaje enfocados en un ámbito ambiental; no obstante, para futuras investigaciones, es posible estudiar a los otros prestadores de servicios turísticos, como los restaurantes y los centros de entretenimiento, entre otros, para reconocer las diversas necesidades del sector turístico en materia de protección ambiental.

\section{REFERENCIAS}

Agle, B. R., Mitchell, R. K. \& Sonnenfeld, J. A. (1999). Who matters to CEOs? An investigation of stakeholder attributes and salience, corporate performance, and CEO values. Academy of Management Journal, 42(5), 507-525.

Aguinis, H. \& Glavas, A. (2012). What we know and don't know about corporate social responsibility: A review and research agenda. Journal of Management, 38(4), 932-968.

Albizu, E. \& Landeta, J. (2001). Dirección estratégica de los recursos humanos: teoría y práctica. Madrid: Ediciones Pirámide. 
Álvarez, M. J., De Burgos, J. \& Céspedes, J. J. (2001). Un análisis exploratorio de las estrategias medioambientales y el contexto organizativo de los hoteles españoles. Cuadernos de Economía y Dirección de la Empresa, 8, 5-32. Azzone, G., Brophy, M., Noci, G., Welford, R. \& Young, W. (1997). A stakeholders' view of environmental reporting. Long range planning, 30(5), 699-709.

Baro, M. (2011). Jerarquización de stakeholders para la construcción del capital social de las organizaciones. Mediaciones Sociales, 9, 135-162.

Barrett, S. (2002). The strategy of treaty negotiation: "broad but shallow" versus "narrow but deep". En Economic Theory for the Environment: Essays in Honour of Karl-Göran Mäler (pp. 26-40). Cheltenham: Edward Elgar Publishing.

Berry, M. A. \& Rondinelli, D. A. (1998). Proactive corporate environmental management: A new industrial revolution. The Academy of Management Executive, 12(2), 38-50.

Best, J. (1987). Rhetoric in claimsmaking. Social Problems, 34(2), 101-121.

Buysse, K. \& Verbeke, A. (2003). Proactive environmental strategies: A stakeholder management perspective. Strategic Management Journal, 24(5), 453-470.
Céspedes Lorente, J., De BurgosJiménez, J. \& Álvarez-Gil, M. J. (2003). Stakeholders' environmental influence. An empirical analysis in the spanish hotel industry. Scandinavian Journal of Management, 19(3), 333-358.

Collins, L. \& Usher, S. (2004). Project pascalea-public and stakeholder consultation in developing high-profile corporate environmental strategy. Corporate Social Responsibility and Environmental Management, 11(2), 95-102.

Delmas, M. \& Toffel, M. W. (2004). Stakeholders and environmental management practices: An institutional framework. Business Strategy and the Environment, 13(4), 209-222.

Dief, M. E. \& Font, X. (2010). The determinants of hotels' marketing managers' green marketing behaviour. Journal of Sustainable Tourism, 18(2), 157-174.

Donaldson, T. \& Preston, L. E. (1995). The stakeholder theory of the corporation: Concepts, evidence, and implications. Academy of Management Review, 20(1), 65-91.

Fassin, Y. (2012). Stakeholder management, reciprocity and stakeholder responsibility. Journal of Business Ethics, 109(1), 83-96.

Fernández, R. \& Nieto, M. (2002). Atributos medioambientales de los stakeholders: un análisis em- 
pírico de la industria manufacturera española. Conferencia presentada en el XVI Congreso nacional, XII Congreso hispano francés: La empresa intangible. Alicante, España.

Fineman, S. \& Clarke, K. (1996). Green stakeholders: industry interpretations and response. Journal of Management Studies, 33(6), 715-730.

Fleming, P., Roberts, J. \& Garsten, C. (2013). In search of corporate social responsibility: Introduction to special issue. Organization, 20(3), 337-348.

Frederick, W. C., Post, J. E. \& Davis, K. (1992). Business and society: corporate strategy, public policy, ethics. Nueva York: McGraw-Hill.

Freeman, R. E. (2010). Strategic management: A stakeholder approach. Cambridge: University Press.

Frooman, J. (1999). Stakeholder influence strategies. Academy of Management Review, 24(2), 191205.

Gilbert, J. A., Stead, B. A. \& Ivancevich, J. M. (1999). Diversity management: A new organizational paradigm. Journal of Business Ethics, 21(1), 61-76.

González Esteban, E. (2012). El modelo de empresa plural desde el enfoque de los skateholders. Recuperado de http://repositori. uji.es/xmlui/bitstream/handle/ $10234 / 68302 / 55510$. pdf? sequence $=1$

Grafé Buckens, A. \& Hinton, A.-F. (1998). Engaging the stakeholders corporate views and current trends. Business Strategy and the Environment, 7(3), 124-133.

Habermas, J.(1989). Teoría de la acción comunicativa: complementos y estudios previos. Madrid: Cátedra.

Hajer, M. A. (1995). The politics of environmental discourse: Ecological modernization and the policy process. Oxford: Oxford University Press.

Hallahan, K. (2001). Inactive publics: The forgotten publics in public relations. Public Relations Review, 26(4), 499-515.

Handfield, R., Sroufe, R. \& Walton, S. (2005). Integrating environmental management and supply chain strategies. Business Strategy and the Environment, 14(1), 1-19.

Hannigan, J. A. (1997). Environmental sociology: A social constructionist perspective. LondresNueva York: Routledge.

Henriques, I. \& Sadorsky, P. (1999). The relationship between environmental commitment and managerial perceptions of stakeholder importance. Academy of Management Journal, 42(1), 87-99. 
Hill, C. W. \& Jones, T. M. (1992). Stakeholder-Agency theory. Journal of management studies, 29(2), 131-154.

Ilinitch, A. Y., Soderstrom, N. S. \& Thomas, T. E. (1999). Measuring corporate environmental performance. Journal of accounting and public policy, 17(4), 383-408.

Ioannides, D. \& Debbage, K. G. (1998). The economic geography of the tourist industry: A supply-side analysis. Londres: Routledge.

Jensen, T. \& Sandström, J. (2011). Stakeholder Theory and Globalization: The challenges of power and responsibility. Organization Studies, 32(4), 473-488.

Johnson, D. B. (1998). Green Businesses: Perspectives from management and business ethics. Society y Natural Resources, 11(3), 259-266.

Klassen, R. D. \& Vachon, S. (2003). Collaboration and evaluation in the supply chain: The impact on plant-level environmental investment. Production and Operations Management, 12(3), 336352.

Kuper D. (2009). Turismo y preservación ambiental: el desarrollo turístico de Península Valdés, Provincia del Chubut. Pasos, 7(1), 85-97.

Lawrence, A. T. \& Weber, J. (2011). Business and Society: Stakehol- ders, Ethics, Public Policy. 13a ed. Nueva York: McGraw-Hill Irwin.

Ledingham, J. A. (2009). A chronology of organization-stakeholder relationships with recommendations concerning practitioner adoption of the relational perspective. Journal of Promotion Management, 14(3-4), 243262.

Lezama, J. L. (2004). La construcción social y política del medio ambiente. México: El Colegio de México.

Llul Gilet, A. (2001). Contabilidad medioambiental y desarrollo sostenible en el sector turístico. (Tesis doctoral inédita). Universitat de les Illes Balears, Palma, España.

MacNaghten, P. \& Urry, J. (2001). Introduction. En P. Macnaghten \& J. Urry (eds.), Bodies of $\mathrm{Na}$ ture (pp. 1-11). Londres: SAGE.

Miller, J. \& Szekely, F. (1995). What is "green"? Environmental Impact Assessment Review, 15(5), 401-420.

Mintzberg, H. (1983). Power in and around Organizations. Vol. 142. Englewood Cliffs: Prentice-Hall.

Mitchell, R. K., Agle, B. R. \& Wood, D. J. (1997). Toward a theory of stakeholder identification and salience: Defining the principle of who and what really counts. Academy of Management Review, 22(4), 853-886. 
Mitchell, R. K., Agle, B. R., Chrisman, J. J. \& Spence, L. J. (2011). Toward a theory of stakeholder salience in family firms. Business Ethics Quarterly, 21(2), 235-255.

Orts, E. W. \& Strudler, A. (2002). The Ethical and environmental limits of stakeholder theory. $\mathrm{Bu}$ siness Ethics Quarterly, 12(2), 215-233.

Parmar, B. L., Freeman, R. E., Harrison, J. S., Wicks, A. C., Purnell, L. \& De Colle, S. (2010). Stakeholder Theory: The State of the Art. The Academy of Management Annals, 4(1), 403-445.

Pfeffer, J. \& Salancik, G. R. (1978). The external control of organizations: A resource dependence perspective. Nueva York: Harper y Row.

Phillips, R. (2003). Stakeholder theo$r y$ and organizational ethics. San Francisco: Berrett-Koehler.

Phillips, R. \& Freeman, R. E. (eds.). (2010). Stakeholders. Cheltenham: Edward Elgar.

Polonsky, M. J., Rosenberger III, P. J. \& Ottman, J. (1998). Developing green products: Learning from stakeholders. Asia Pacific Journal of Marketing and Logistics, 10(1), 22-43.

Raufflet, E., Lozano, J. F., Barrera, E. \& De la Torre, C. G. (2012). Responsabilidad social empresarial. México D. F.: Pearson.

Rawlins, B. L. (2006). Prioritizing Stakeholders for Public Rela- tions. Recuperado de http://painepublishing.com/wp-content/ uploads/2014/03/prioritizingstakeholders-Rawlins.pdf

Revenga, A. (2007). Francis Fukujama: confianza y progreso en el fin de la historia. En R. Alcoberro (coord.). Ética, economía y empresa (pp. 191-200). Barcelona: Gedisa.

Rodríguez, M. Á. \& Ricart, J. E. (1997). Estrategia medioambiental: principales factores y fuerzas medioambientales. Harvard Deusto Business Review, 78, 5673.

Sharma, S. \& Henriques, I. (2005). Stakeholder influences on sustainability practices in the canadian forest products industry. Strategic Management Journal, 26(2), 159-180.

Stead, J. G. \& Stead, E. (2000). EcoEnterprise strategy: Standing for sustainability. Journal of Business Ethics, 24(4), 313-329.

Stiglitz, J. E. (2002). Globalization and its discontents. Nueva York: W. W. Norton.

Stiglitz, J. E. (2007). Making globalization work. Nueva York: W. W. Norton.

Thomas, L. M. (1992). The business community and the environment: An important partnership. Business Horizons, 35(2), 21-24.

Tilt, C. A. (1997). Environmental policies of major companies: Australian evidence. The Bri- 
tish Accounting Review, 29(4), 367-394.

Vargas, E. E., López, L. J. \& Martínez, R. S. (2014). La dimensión ambiental en las preferencias de consumo del turista. Caso Cancún. Revista de Administração da Unimep, 12(2), 42-58.

Vargas, E. E., Zizumbo, L., Viesca, F. C. \& Del Carmen Serrano, R. (2011). Gestión ambiental en el sector turístico mexicano.
Efectos de la regulación en el desempeño hotelero. Cuadernos de Administración, 24(42), 183204.

Welford, R. \& Gouldson, A. (1993). Environmental management \& business strategy. Londres: Pitman Publishing Limited.

Wood, D. J. (1991). Corporate social performance revisited. Academy of Management Review, 1 , 691-718. 